\title{
The use of calcium and vitamin D in the management of osteoporosis
}

\author{
John A Sunyecz \\ MenopauseRx, Inc., Uniontown, PA, \\ USA
}

Correspondence: John A Sunyecz President, MenopauseRx, Inc., 77 Wiggins Lane, Uniontown, PA I540I, USA

$\mathrm{Tel}+\mathrm{I} 7243223665$

Fax +I 7244388856

Email sunyecz@menopauserx.com

\begin{abstract}
Osteoporosis poses a significant public health issue, causing significant morbidity and mortality. Calcium and vitamin D utilization in the optimization of bone health is often overlooked by patients and health care providers. In addition, the optimal standard of care for osteoporosis should encompass adequate calcium and vitamin D intake. Dietary intake or supplementation with calcium and vitamin $\mathrm{D}$ will be reviewed, including recent recommendations for increased vitamin D intake. Compliance to calcium and vitamin D therapy is paramount for effective prevention of osteoporotic fractures. A recently released algorithm (FRAX) estimating absolute fracture risk allows the health care provider to decide when pharmacologic therapy is warranted in addition to calcium and vitamin D. When pharmacologic therapy is advised, continued use of calcium and vitamin D is recommended for optimal fracture risk reduction. A 'bricks and mortar' analogy is often helpful when counseling patients and this analogy will be explained. This manuscript reviews relevant data related to calcium and vitamin D use for patients at risk for fracture due to bone loss.
\end{abstract}

Keywords: osteoporosis, calcium, vitamin D, osteopenia, hip fracture, bone

\section{Osteoporosis overview}

The skeletal disease of bone thinning and compromised bone strength, osteoporosis, continues to be a major public health issue as the population ages. This disease is characterized by bone fragility and an increased susceptibility to fractures, especially of the spine and hip, although any bone can be affected. It is estimated that over 10 million Americans over the age of 50 have osteoporosis. Risk for osteoporosis has been reported in people of all ethnic backgrounds. An additional 34 million have reduced bone mass, called osteopenia, which puts them at higher risk for fractures later in life (USDH 2004). The risk of fracture from osteoporosis increases with age. There are approximately 1.5 million osteoporotic fractures per year reported in women and men in the US, including over 300,000 hip fractures. As the population ages, this number will probably increase. The US Surgeon General estimates that one out of every two women over the age of 50 will have an osteoporosis-related fracture in their lifetime. In addition, $20 \%$ of those affected by osteoporosis are men with $6 \%$ of white males over the age of 50 suffering a hip fracture. It is estimated that the national direct care costs for osteoporotic fractures is US\$12.2 to 17.9 billion per year in 2002 dollars, with costs rising. This cost is comparable to the Medicare expense for coronary heart disease (\$11.6 billion) (Thom et al 2006).

Since bone loss occurs without symptoms, osteoporosis is often considered a 'silent disease'. As deterioration of bone tissue mounts and disruption of bone architecture occurs, the bone becomes so weak that a relatively minor bump or fall causes a fracture or vertebrae to collapse. The resulting fracture may lead to loss of mobility and independence, with 25\% requiring long term care (NAMS 2006). Fractures caused by either osteoporosis or low bone mass can lead to chronic pain, disability, as well as 
psychological symptoms, including depression. A woman's risk of hip fracture is equal to her combined risk of breast, uterine and ovarian cancer). Unfortunately, approximately $24 \%$ of patients with hip fractures over the age of 50 will die in the year following the fracture (NOF 2008).

Fortunately, the importance of this debilitating bone disease is being recognized. President Bush has declared 2002-2011 as the Decade of the Bone and Joint. Important advances have been made to understand the disease process and help create therapies to treat the condition. Bone health is optimized by creating an environment to achieve peak bone mass during adolescence, maintenance of healthy bone throughout life and prevention of bone loss with aging. Health care providers are vital to identify patients at risk for bone loss and diagnose bone thinning so that prevention and treatment strategies are effective.

The US Surgeon General has outlined a 'pyramid approach' to treating bone diseases. Prevention of falls with maintenance of bone health through adequate calcium, vitamin D, and physical activity represent the base of the pyramid for all individuals, including those with bone disease. The second tier of this pyramid relates to identifying and treating secondary causes of osteoporosis. Lastly, the third tier revolves around pharmacotherapy (USDH 2004).

Calcium and vitamin D have long been recognized as important and required nutrients for bone health and maintenance. The continuation of calcium and vitamin $\mathrm{D}$ in a patient with bone loss is critical for optimal care. Unfortunately, 90\% of women may not be getting enough calcium and over $50 \%$ of women treated for bone loss have inadequate vitamin D levels (NDC 2004; Holick et al 2005). Currently, there are a number of pharmacologic treatments for osteoporosis which provide improvements in bone mass and reduction in fracture risk. These treatments have been studied where adequate calcium and vitamin D supplementation had been achieved. Therefore, their use is predicated on proper calcium and vitamin D therapy. The goal of this manuscript is to review data related to calcium and vitamin $\mathrm{D}$ in the management of osteoporosis.

\section{Calcium}

\section{Nutrition}

Peak bone mass is usually achieved by age 30; therefore, physical activity and obtaining the recommended doses of calcium and vitamin D in adolescence and young adulthood will ensure peak bone mass development (Table 1) (IOM 1997). Calcium is an essential element in the human body and is necessary to many cell functions. Calcium is
Table I Recommended dietary intake of calcium and vitamin D. Adapted with permission from Institute of Medicine, Food and Nutrition Board (1997)

\begin{tabular}{lll}
\hline Age (years) & Calcium (mg/day) & Vitamin D (IU/day) \\
\hline $4-8$ & 800 & 200 \\
$9-13$ & 1300 & 200 \\
$14-18$ & 1300 & 200 \\
$19-30$ & 1000 & 200 \\
$31-50$ & 1000 & 200 \\
$51-70$ & 1200 & 400 \\
$\geq 70$ & 1200 & 600 \\
\hline
\end{tabular}

not only important to bone health, but it is also essential for neuromuscular activity, blood coagulation, and normal cardiac function. It is a vital component of bone architecture and is required for deposition of bone mineral throughout life. Although the body stores more than $99 \%$ of its calcium in the bones and teeth, it is also found in the extracellular fluid (ECF) or plasma. It is the levels of plasma calcium that dictate calcium balance. If the plasma level decreases, bone resorption increases to restore plasma levels. Adequate intake of calcium is necessary to maintain this balance. Calcium is absorbed in the small intestines with the aid of vitamin D (Bringhurst et al 2005). Excretion of calcium is primarily through the kidneys, although there is minor fecal loss.

The best way to meet the daily dietary requirement is through the intake of high calcium containing foods. Dairy products are the best sources of calcium due to their high elemental calcium content, high absorptive rate, and relative low cost. Dietary sources of calcium include dairy products (milk, cheese, yogurt) and some green vegetables. Each daily dairy serving consumed contains approximately 300 milligrams. A serving size of dairy equals one cup ( 8 ounces) of milk, one cup of yogurt or one to 1.5 ounces of cheese. Therefore, each daily dairy serving multiplied by $300 \mathrm{mg}$ would provide an estimated total elemental calcium consumption (IOM 1997). Mineral waters enriched with calcium are another source of dietary calcium. A recent study showed that high-calcium mineral waters had absorbabilities equal to milk calcium or slightly better and may provide useful quantities of bio-available calcium (Heaney 2006).

The individuals who do not obtain enough calcium from foods should take a supplement to meet these guidelines. Evidence suggests that the average American does not meet the lower end of the recommended daily calcium intake. The average women over the age of 40 has a calcium intake of less than half the amount recommended for postmenopausal 
women. In one study, $82 \%$ of patients with osteoporosis were taking less than the recommended $1000 \mathrm{mg}$ daily (Black et al 1996). This highlights the need for calcium supplementation in all patient populations. In fact, patients with osteoporosis are more likely to have a history of inadequate dietary calcium intake (NIH 1994).

\section{Supplements}

In order to assure adequate calcium intake, a number of calcium supplements are readily available. The two most common and well-studied calcium supplements are calcium carbonate and calcium citrate. Both supplements have been shown to be equally well absorbed when taken with food (Heaney et al 2001). In the past, it was assumed that gastric acid secretion and gastric acidity played a critical role in the intestinal absorption of calcium. A randomized crossover trial demonstrated that the proton pump inhibitor, omeprazole, markedly decreased fractional calcium absorption from calcium carbonate when ingested by elderly women after an overnight fast on an empty stomach (O'Connell et al 2005). In addition, a case control study reported that long-term proton pump inhibitor therapy, particularly at high doses, was associated with an increased risk of hip fracture (Yang et al 2006). Research appears to support that calcium absorption can be ensured by ingestion with food. Recker (1985) reported normal absorption of calcium carbonate supplements when taken with a meal, even in achlorhydric patients. A study to evaluate the role of gastric acid on calcium absorption reported that a large does of cimetidine, which reduced gastric acid secretion, had no effect on calcium absorption from carbonate or citrate sources. In addition, calcium carbonate absorption was the same whether gastric contents were maintained at a $\mathrm{pH}$ of 7.4 or 3.0 (Bo-Linn et al 1984).

Calcium carbonate supplements have the highest percentage of elemental calcium among the calcium salts (Table 2). Calcium carbonate contains $40 \%$ elemental calcium compared to $21 \%$ found in calcium citrate, $13 \%$ found in calcium lactate, and $9 \%$ found in calcium gluconate (Weisman 2005).

Table 2 Percentage of elemental calcium in common calcium salts. Adapted with permission from Weisman (2005)

\begin{tabular}{ll}
\hline Calcium salt & Elemental calcium (\%) \\
\hline Carbonate & 40 \\
Tricalcium phosphate & 38 \\
Citrate & 21 \\
Lactate & 13 \\
Gluconate & 9 \\
\hline
\end{tabular}

From a patient perspective, this translates into fewer calcium carbonate tablets required to achieve optimal intake on a daily basis. Quality calcium products should come from a reputable manufacturer who can provide adequate absorbability and bioavailability data. Given equivalent bioavailability of carbonate and citrate supplements, the cost benefit analysis favors the less expensive carbonate products. In fact, a leading calcium citrate product (Citracal) was reported to cost 1.5-1.8 times a leading calcium carbonate (OsCal) product when comparing grams of elemental calcium (Heaney et al 2001).

To ensure optimal absorption, patients should be counseled to take their calcium supplements with meals in divided doses. Since the GI tract can only absorb 500 to $600 \mathrm{mg}$ of calcium at one time, supplements should be spaced by at least four to five hours to achieve the recommended intake (Heaney et al 1975).

Side effects from calcium supplements are few. Although GI side effects are often mentioned, the recently reported Women's Health Initiative (WHI) did not reveal any difference between the calcium/vitamin D group and placebo group in the rate of adverse GI events, such as gas, bloating, or constipation (Jackson et al 2006). Clinically, it is worthwhile to recommend initiating a calcium/vitamin D supplement at a lower dose with gradual titration to the target intake amount over 1-2 months in patients that describe GI symptoms.

\section{Drug interactions}

Calcium supplements have the potential to interact with several prescription and over the counter medications. Calcium supplements may decrease levels of the drug digoxin. The interaction between calcium and vitamin D supplements and digoxin may also increase the risk of hypercalcemia. Calcium supplements also interact with fluoroquinolones, levothyroxine, antibiotics in the tetracycline family, and phenytoin. In all of these cases, calcium supplements decrease the absorption of these drugs when the two are taken at the same time (Jellin et al 2000; Shannon et al 2000).

Thiazide, and diuretics similar to thiazide, can interact with calcium and vitamin D supplements to increase the chances of developing hypercalcemia and hypercalciuria (Jellin et al 2000). Aluminum and magnesium antacids can both increase urinary calcium excretion. Mineral oil and stimulant laxatives can both decrease dietary calcium absorption (Jellin et al 2000).

In general, bisphosphonate drugs are poorly absorbed from the GI tract and can bind calcium. Therefore, bisphosphonate drugs should be taken on an empty stomach with a 
30-60 minute post-dose fast. To ensure adequate absorption, it is prudent to avoid taking calcium supplements around the dose of oral bisphosphonates.

Often, the physician identifying and treating the patient for bone loss is not the same health care provider treating other acute and chronic medical conditions. Therefore, it is imperative to understand the pharmacology of the medications prescribed to patients and the potential interactions with calcium and vitamin D supplements.

\section{Food interactions}

Achieving optimal calcium intake requirements through diet alone can prove even more difficult in certain populations, such as vegetarians, those who are lactose intolerant and those with calorie-restriced diets. In addition, there are several substances that can interfere with the body's ability to use calcium, including oxalate, protein, phytate, and caffeine.

Foods with high amounts of oxalate and phytate reduce the absorption of calcium contained in those foods. Spinach, rhubarb and beet greens are examples of foods that are high in oxalate. While these foods can be an important part of a healthy diet, they are not good sources of calcium.

Legumes, such as pinto beans, navy beans, and peas, are high in phytate. Wheat bran is also high in phytate. Therefore, it is recommended to avoid taking calcium supplements with oxalate- or phytate-rich foods to ensure adequate absorption.

\section{Kidney stones}

Kidney stones are crystallized deposits of calcium and other minerals in the urinary tract. Calcium oxalate stones are the most common form of kidney stones in the US. High calcium intakes or high calcium absorption were previously thought to contribute to the development of kidney stones. Other factors such as high oxalate intake and reduced fluid consumption appear to be more of a risk factor in the formation of kidney stones than calcium in most individuals.

A $17 \%$ increase in kidney stone formation was seen in the WHI study. However, this increase in kidney stone formation is contrary to a number of prior studies that showed no increased risk or a decreased risk of kidney stone formation; further study is warranted. Borghi and colleagues (2002) reported a $50 \%$ reduction in stone recurrence in men with a history of kidney stones who were assigned a calcium diet of $1200 \mathrm{mg} /$ day compared to a low calcium group. Data from the Nurses Health Study who were followed for 12 years revealed that women with greater than $1000 \mathrm{mg} /$ day calcium had lower risks of kidney stones compared to women with daily intake of less than $500 \mathrm{mg}$ /day (Curhan et al 1997).

\section{Vitamin D}

\section{Nutrition}

Vitamin D is an important nutrient in the maintenance of bone health. The primary functions of vitamin $\mathrm{D}$ are the regulation of intestinal calcium absorption and the stimulation of bone resorption leading to the maintenance of serum calcium concentration (Reid et al 2003). Sources of vitamin $\mathrm{D}$ include sunlight, diet, and supplements. The majority of Americans do not achieve adequate vitamin D levels. In fact, it is estimated that $90 \%$ of adults between 51 and 70 years of age do not get enough vitamin $\mathrm{D}$ from their diet (Moore et al 2004).

Sunlight exposure, or ultraviolet B (UVB) radiation, is absorbed by the 7-dehydrocholesterol that resides in the skin to form previtamin D3. Previtamin D3, an unstable compound, is quickly converted to vitamin D3 via heat (MacLaughlin et al 1982). Vitamin D3 moves out into the extracellular space and is drawn into the capillaries by vitamin D-binding protein (DBP) (Holick 2005a). Once in the capillaries, the vitamin $\mathrm{D}$ is transported to the liver where it undergoes hydroxylation to form 25-hydroxyvitamin $\mathrm{D}$ [25(OH)D]. 25-hydroxyvitamin $\mathrm{D}$ is again bound by DBP and taken to the kidney where it is transported and released into the renal tubule cell and hydroxylated to form 1,25-dihydroxyvitamin D [1,25(OH)2D] (DeLuca 2004). This is the biologically active form of vitamin $\mathrm{D}$, which is responsible for calcium homeostasis.

Dietary sources of vitamin D are absorbed into the lymphatic system via chylomicrons, where they enter the circulation and are bound by DBP (Bouillon 2001). From here, they are taken to the liver and kidneys, as explained above, for the formation of the active form of vitamin $\mathrm{D}$ $[1,25(\mathrm{OH}) 2 \mathrm{D}]$.

\section{Vitamin D deficiency}

In vitamin D deficiency states, decreased calcium absorption occurs from the intestines, causing increased osteoclast production, which enhances the mobilization of calcium from the bone. During periods of decreased dietary intake, $1,25(\mathrm{OH}) 2 \mathrm{D}$ interacts with receptors in osteoblasts, ultimately leading to increased formation of osteoclasts (Holick 2004a). The mature osteoclast then releases enzymes to breakdown bone matrix ultimately releasing calcium and other minerals into the circulation (Holick 2005b). If the serum free calcium level remains low, the parathyroid gland 
is stimulated (Holick 2005a). Release of parathyroid hormone (PTH) causes increased renal reabsorption of calcium and also stimulates osteoclast production, leading to increased serum levels of calcium. If vitamin D deficiency is not corrected, calcium continues to be pulled from the bone and rickets can occur in children, while osteomalacia and osteoporosis can occur in adults.

\section{Vitamin D sources}

Sunlight is the most common source of vitamin D. Serum $25(\mathrm{OH}) \mathrm{D}$ levels are lower in individuals who use sunscreens and in those with pigmented skin. A practical and common recommendation for adequate vitamin $\mathrm{D}$ from sunlight is five to 15 minutes of sun exposure from the hours of $10 \mathrm{AM}$ to $3 \mathrm{PM}$ in the spring, summer, and autumn at least two times per week to the face, arms, hands, or back. This is usually enough for people with most skin types to maintain adequate vitamin D levels (Holick 2004b). After this exposure, sunscreen could be applied to limit solar skin damage. Therefore, it is important to remember that while harmful UV rays from sunlight can increase skin cancer risks, blocking these UV rays can predispose individuals to vitamin $\mathrm{D}$ deficiency.

If sufficient sunlight is not obtained, dietary sources of vitamin D can be utilized. Dietary sources of vitamin D include fatty fish such as salmon, mackerel, and sardines which provide 300 to 600 units/3.5 ounces, egg yolks which provide 20 units/yolk, and cod liver oil which provides 400 units/teaspoonful. The most common dietary source of vitamin D is found in fortified foods such as milk, orange juice, and some cereals which provide about 100 units per serving (Tangpricha et al 2003; Holick 2004a).

Due to the relative lack of vitamin D-containing foods, supplements of vitamin D are often necessary to achieve an adequate intake. The National Osteoporosis Foundation (NOF) recommends an intake of 800 to 1000 international units (IU) of vitamin D3 per day for adults over age 50 (NOF 2008). The safe upper limit for vitamin D intake for the general adult population was set at 2,000 IU per day in 1997 (SCSEDR 1997). Recent evidence indicates that higher intakes are safe and that some elderly patients will need at least this amount to maintain optimal 25(OH)D levels (NOF 2008).

Dietary sources of vitamin D can come from plants (ergocalciferol or vitamin D2) or animals (cholecalciferol or vitamin D3). Supplements can come from either source. A review of the medical and lay press appears to conclude that vitamin D2 is less effective than vitamin D3 in maintaining vitamin D status. A recent placebo controlled study compared intakes of 1000 units of D2 and 1000 units of D3 for 11 weeks at the end of winter. Interestingly, 67\% of participants were vitamin D deficient at the start of the study. The authors concluded that 1000 units of vitamin D2 daily was as effective as 1000 units of vitamin D3 in maintaining serum 25-hydroxyvitamin D levels (Holick et al 2008). Therefore, vitamin D2 and D3 supplements should be considered comparable and of equal potency.

Since higher intakes of vitamin $\mathrm{D}$ can reduce bone resorption and subsequent bone loss, it appears that the recommended intake of vitamin D is in need of revised requirements, especially in older individuals. In an extensive review of the vitamin D literature, Vieth (1999) states that the daily reference intake should be 800-1000 units per day based upon bone density measurements and fracture prevention in the elderly. A recent report found that levels of $25(\mathrm{OH}) \mathrm{D}$ less than $10 \mathrm{ng} / \mathrm{ml}$ was linked to a $78 \%$ increase risk of falling in men and women over the age of 65 (Snijder et al 2006).

Fifty-two percent of North American women receiving therapy to prevent or treat osteoporosis were found to be vitamin D deficient after measuring serum 25(OH)D levels (Holick et al 2005). Furthermore, a survey of childbearing women in the US found $41 \%$ of African American women between 15 and 49 years of age to be vitamin $\mathrm{D}$ deficient at the end of winter (Nesby-O'Dell et al 2002). Lastly, 4\% of Caucasian women were found to be vitamin $\mathrm{D}$ deficient at the end of summer.

Vitamin D serum levels can be measured to determine vitamin D stores. The value that is measured is $25(\mathrm{OH}) \mathrm{D}$ because this compound has the longest half life (two weeks) and it correlates with secondary hyperparathyroidism, rickets, and osteomalacia (unmineralized bone) (Holick 2006). The normal range of vitamin D $(25(\mathrm{OH}) \mathrm{D})$ is $10-55 \mathrm{ng} / \mathrm{ml}$ (25-137.5 nmol/L) (Weaver and Fleet 2004). The desired adult level of vitamin D (25(OH)D) is $30 \mathrm{ng} / \mathrm{ml}(75 \mathrm{nmol} / \mathrm{L})$ or higher (NOF 2008).

\section{Osteoporosis} Diagnosis

Recently, the NOF released its new Clinician's Guide to Prevention and Treatment of Osteoporosis (NOF 2008). These guidelines are described as a major breakthrough in the way health care providers evaluate and treat patients with low bone mass or osteoporosis and the risk of fractures. The guidelines allow specific recommendations to many races, including Caucasian postmenopausal women, as well as African-American, Asian, Latina, and other postmenopausal women. It also addresses men aged 50 and older for the first time. 
The most common clinical tool to diagnose osteoporosis and predict fracture risk is a bone mineral density (BMD) test. A measurement of bone density is often considered when it will help guide decisions regarding treatment to prevent osteoporotic fractures. The NOF recommends testing all women age 65 and older and men age 70 and older, regardless of clinical risk factors. Other indications for BMD testing include: (NOF 2008)

- Younger postmenopausal women and men aged 50-70 about whom you have concern based on their clinical risk factor profile.

- Women in the menopausal transition if there if a specific risk factor associated with increased fracture risk such as low body weight, prior low-trauma fracture, or high risk medication.

- Adults who have a fracture after age 50 .

- Adults with a condition (eg, rheumatoid arthritis) or taking a medication (eg, glucocorticoids, $\geq 5 \mathrm{mg}$ /day for $\geq 3$ months) associated with low bone mass or bone loss.

- Anyone being considered for pharmacologic therapy for osteoporosis.

- Anyone being treated for osteoporosis, to monitor treatment effect.

- Anyone not receiving therapy in whom evidence of bone loss would lead to treatment.

- Postmenopausal women discontinuing estrogen therapy should be considered for bone density testing.

The updated NOF guidelines include the use of a new algorithm on absolute fracture risk, called FRAX by the World Health Organization (WHO). The FRAX algorithm takes into account not only BMD at the hip but also nine specific clinical risk factors for osteoporosis and related fractures. This algorithm estimates the 10-year hip fracture probability and 10-year all major osteoporosis-related fracture probability. Treatment is recommended for patients with hip or vertebral (clinical or morphometric) fractures, as well as those with BMD T-scores $\leq-2.5$ at the femoral neck, total hip, or spine by DXA, after appropriate evaluation.

Treatment guidelines help the clinician decide which patients with osteopenia warrant pharmacologic treatment. Treatment is recommended for postmenopausal women and in men age 50 and older with low bone mass (T-score -1 to -2.5 , osteopenia) at the femoral neck, total hip, or spine and 10 -year hip fracture probability $\geq 3 \%$ or a 10 -year all major osteoporosis-related fracture probability of $\geq 20 \%$ based on the US-adapted WHO absolute fracture risk model.

Menopause often leads to increases in bone loss with the most rapid rates of bone loss occurring during the first five years after menopause (Gallagher et al 1987). A drop in estrogen production after menopause results in increased bone resorption, and decreased calcium absorption (Gallagher et al 1980; Breslau 1994). Annual decreases in bone mass of $3 \%-5 \%$ per year are often seen during the years immediately following menopause, with decreases less than $1 \%$ per year seen after age 65 (Daniels 2001). Postmenopausal hormone therapy can decrease the incidence of osteoporosis and reduce fracture risk. However, since many women have elected to discontinue or avoid hormone therapy after menopause, it is imperative for health care providers to actively identify those women at risk for bone thinning and fracture. Counseling is especially prudent regarding weight bearing exercise and calcium/vitamin D intake during the perimenopause transition.

\section{Treatment considerations Calcium studies}

Calcium supplementation has shown effectiveness for preventing osteoporotic fractures in postmenopausal women. A meta-analysis reviewed the literature from 1966 to 1997 assessing the effectiveness of calcium supplementation for the prevention of osteoporotic fractures in postmenopausal women. Only studies with fracture outcomes were eligible. This analysis revealed relative risk reductions between $25 \%$ to $70 \%$ for osteoporotic fractures. Most trials reported an approximate $30 \%$ fracture risk reduction with an intake of approximately $1000 \mathrm{mg}$ /day of elemental calcium (Cummings and Nevitt 1997).

Over 18 months, a trial of elderly French women found that the number of hip fractures was reduced by $43 \%$ and nonvertebral fractures was reduced by $32 \%$ among women treated with calcium $(1200 \mathrm{mg} /$ day $)$ and vitamin D (800 units/day) than among those who received placebo (Chapuy et al 1992).

Dawson-Hughes and colleagues (1990) reported that bone loss is reduced in healthy, older postmenopausal women by increasing calcium intake from $400 \mathrm{mg}$ /day to $800 \mathrm{mg} /$ day. In addition, postmenopausal women (within three to six years of menopause) supplemented with $1700 \mathrm{mg} /$ day of calcium had reduced bone loss from the femoral neck and improved calcium balance in a study of 118 healthy white women (Aloia et al 1994). Another study revealed that dietary supplementation of calcium and vitamin $\mathrm{D}$ in men and women over 65 years of age could reduce bone loss in the femoral neck and spine while reducing the incidence of nonvertebral fractures over three years (Dawson-Hughes et al 1997). 
A meta-analysis of 15 trials with 1806 study participants found that calcium alone caused a positive mean percentage BMD change from baseline of $2.05 \%$ for total body bone density, $1.66 \%$ at the lumbar spine, $1.6 \%$ at the hip, and $1.9 \%$ at the distal radius. The data also showed a trend toward reduction in vertebral fractures, with relative risk reduction of 0.79 ( $95 \%$ CI 0.54 to 1.09 ). However, the risk reduction for nonvertebral fractures was unclear $(\mathrm{RR}=0.86(95 \%$ CI 0.43 to 1.72 ) (Shea et al 2004).

The recent RECORD trial studied 5292 people over 70 years of age to evaluate the effectiveness of calcium and/or vitamin D supplements for the secondary prevention of fractures. Follow-up was between 24 and 62 months. The groups studied did not reveal significant reductions in future fractures. However, compliance with the supplements was a significant problem with this study. At 24 months, only $54.5 \%$ of subjects were still taking the supplement (Grant et al 2005).

A more recent meta-analysis of 29 studies, with over 63,000 individuals analyzed, looked at calcium and calcium in combination with vitamin D trials for use in prevention of fracture and bone loss was recently reported. The authors concluded that treatment was associated with a $12 \%$ risk reduction in fractures of all types. Trials with higher compliance revealed significant risk reduction compared to studies with lower compliance. In the 8 studies with greater than $80 \%$ compliance, a $24 \%$ risk reduction for all fractures was identified. The author's conclusions were that calcium, or calcium in combination with vitamin D supplementation, was effective in the preventive treatment of osteoporosis in people aged 50 years or older. It appeared that the best effect was seen with minimum doses of $1200 \mathrm{mg}$ of calcium and 800 units of vitamin D daily (Tang et al 2007).

Supplementation with vitamin D in individuals with severe calcium deficiency did not lead to bone sparing. However, calcium repletion in study subjects with marginal vitamin D levels resulted in bone sparing. Therefore, optimal intake of vitamin D is influenced by calcium intake. Optimal bone health requires both a high dietary calcium intake and high vitamin D intake (Weaver and Fleet 2004). Counseling patients that many calcium supplements also contain vitamin $\mathrm{D}$ is one convenient mechanism by which adequate supplementation can be achieved.

A recent study from the WHI reported results regarding calcium and vitamin D supplementation and the risk of fractures. This study randomly assigned over 36,000 healthy postmenopausal women to receive daily doses of $1000 \mathrm{mg}$ calcium carbonate and 400 units of vitamin D or placebo, with a seven-year follow up with respect to fractures and BMD. The conclusions drawn from the study revealed a small but significant improvement in hip bone density, but no significant reduction in hip fracture. This trial was well conducted but left many questions unanswered. Pertinent findings within this study were that women who were adherent to their study regimen (those who took over $80 \%$ of their study calcium/vitamin D) had a significant $29 \%$ reduction in hip fracture. Women aged 60 and over (those at highest risk of fracture) had a significant $21 \%$ reduction in hip fracture risk.

Sixty-four percent of women in the placebo group had a daily calcium intake from diet and supplements of at least $800 \mathrm{mg}$ and $42 \%$ had a daily vitamin D intake of at least 400 units. In addition, the study participants had average daily calcium intakes of $1150 \mathrm{mg}$ before the study began, which is nearly double the national average. Therefore, this intake may certainly reduce the chances of detecting a benefit of additional calcium and vitamin D therapy in this study (Jackson et al 2006).

The data from these studies indicate a vital role for calcium and vitamin $\mathrm{D}$ related to optimal bone health. It is imperative that calcium and vitamin D counseling be conducted throughout life. Ensuring peak bone development via adequate nutritional intake during adolescence along with continued repeated discussions throughout a person's life can significantly limit morbidity and mortality from osteoporosis. Poor compliance is a major obstacle in many medical regimens. Based on evidence presented, calcium and vitamin D supplementation compliance is vital to ensuring optimal risk reduction and fracture prevention.

\section{Vitamin D studies}

Vitamin D has been extensively studied regarding its impact on fracture risk reduction. In fact, vitamin D deficiency has been associated with greater incidence of hip fracture in many populations, including postmenopausal women (Chapuy et al 1992; Dawson-Hughes et al 1995). In a detailed review by LeBoff and colleagues (1999) of women with osteoporosis who were hospitalized due to hip fractures, $50 \%$ were found to have signs of vitamin D deficiency.

Reasons for an increased risk of fracture associated with vitamin D deficiency are numerous. Inability to absorb adequate amounts of calcium for optimal bone health, as well as an increased susceptibility of falling, impaired muscle strength, and increased rates of bone loss can increase the risk of fracture (Lips 2001). It has been reported that lower levels of vitamin D are independently associated with an increased 
risk of falling in the elderly. In fact, supplementation with vitamin $\mathrm{D}$ has been shown to improve musculoskeletal function and reduce the risk of falling in elderly women (Bischoff et al 2003). Human muscle contains vitamin D receptors that may lead to increasing muscle strength and improving stability (Snijder et al 2006). The combination of 700 units/ day of vitamin D and $500 \mathrm{mg}$ of calcium (obtained through supplementation in addition to diet) was shown to reduce falls by as much as $65 \%$ over three years in less active women over the age of 65 (Bischoff-Ferrari et al 2006).

It appears that vitamin $\mathrm{D}$ supplementation may prove more beneficial in reducing falls and increasing bone density in elderly women who have suffered a hip fracture when given with calcium (Harwood et al 2004). The Decalyos II study examined the effect of calcium and vitamin D supplementation over two years in a group of elderly women who were able to walk indoors with a cane or walker. Results suggested that such supplementation could reduce the risk of hip fractures in this population (Chapuy et al 2002).

All vitamin D trials from 1966 to 1999 for postmenopausal osteoporosis were evaluated in a meta-analysis to review the effect of vitamin D on bone density and fractures. Twenty-five trials that randomized women to vitamin $\mathrm{D}$ with or without calcium and measured bone density or fracture for at least one year were chosen. The results revealed a $37 \%$ reduction in vertebral fractures (Papadimitropoulos et al 2002).

A Cochrane Database review reported that vitamin D alone showed no statistically significant effect on hip fracture, vertebral fracture, or any new fracture. Vitamin D with calcium marginally reduced hip fractures and nonvertebral fractures, but there was no evidence of effect of vitamin $\mathrm{D}$ with calcium on vertebral fractures. Compliance to the supplements was not reported. The effect appeared to be restricted to those living in institutional care. There was no evidence that vitamin $\mathrm{D}$ increased gastrointestinal symptoms or renal disease. The conclusion was that frail older people confined to institutions may sustain fewer hip and other nonvertebral fractures if given vitamin $\mathrm{D}$ with calcium supplements. Further investigation regarding dose, frequency, and route of administration of vitamin D in older people was recommended (Avenell et al 2005).

Another meta-analysis of randomized, controlled fracture prevention trials with vitamin D reported that oral vitamin D supplementation between 700 to 800 units per day appeared to reduce the risk of hip and any nonvertebral fractures in ambulatory or institutionalized elderly persons. An oral vitamin D dose of $400 \mathrm{IU} / \mathrm{d}$ was not sufficient for fracture prevention. A vitamin D dose of 700 to 800 units per day reduced the relative risk of hip fracture by $26 \%$ and any nonvertebral fracture by 23\% (Bischoff-Ferrari et al 2005). Subsequently, an enhanced meta-analysis was done to define the need for additional calcium supplementation in individuals receiving vitamin D for the prevention of hip fractures. Findings suggested that oral vitamin D appears to reduce the risk of hip fractures only when calcium supplementation is added (Boonen et al 2007).

\section{Pharmacological treatment}

Currently, there are a number of prescription therapeutic options for the prevention and treatment of osteoporosis. The antiresorptive medications are the largest group and include bisphosphonates (Actonel, Boniva, Fosamax, Reclast), selective estrogen receptor modulators (Evista), hormone therapy and calcitonin (Miacalcin). Teriparatide is a parathyroid hormone derivative that acts as an anabolic agent on bone and is indicated for post-menopausal women with osteoporosis who are at high risk of fracture (Eli Lilly 2008). A review of studies that led to the FDA approval of prescription medications used for the treatment of osteoporosis found that $80 \%$ of studies had been performed under conditions where a certain minimum calcium and vitamin D intake was ensured (Sunyecz and Weisman 2005). Therefore, one cannot conclude that these prescription medications would be as effective in calcium and vitamin D deficient patients. Unfortunately, calcium use during osteoporosis therapy is often under-utilized by patients and not appropriately counseled by physicians. In 1994, $43 \%$ of osteoporosis visits had some form of counseling for calcium. By 2000, this proportion had declined to $29 \%$ and fell to $23 \%$ by 2004 (Stafford et al 2005).

An effective tool that can be used to help patients understand the importance of calcium and vitamin D when using prescription medications for bone loss is the 'brick and mortar' analogy. In the analogy, reference is made to building a strong structural foundation. The antiresorptive medication serves as the 'bricks', while adequate calcium and vitamin D serve as the 'mortar'. Without both 'brick' and 'mortar', the structure (healthy bone) could not be achieved.

Oral bisphosphonate medications must be taken on an empty stomach. Package inserts for oral bisphosphonate caution that calcium supplements may interfere with bisphosphonate absorption and should be taken at a different time of day. Calcium citrate products can be taken on an empty stomach or with food, while calcium carbonate products should be taken with meals. Therefore, patients (especially calcium citrate users) need to be counseled to avoid taking their calcium supplements with bisphosphonates. 
It is imperative for the health care provider to be aware that adequate calcium and vitamin D stores must be present to allow pharmacologic treatments for osteoporosis to be effective.

\section{Summary}

Bone loss and osteoporosis with the resultant increase in fracture risk should be major concerns for patients and health care providers. As the population ages, the long term effects of osteoporosis including pain, loss of independence and institutionalized care will become more prevalent. Efforts to prevent bone loss and osteoporosis should start with proper education about a healthy lifestyle, including optimal calcium and vitamin D and exercise in adolescence. This education should continue throughout life, with emphasis during times of increased bone loss such as the menopause transition.

This paper reviews the cornerstone of bone health; calcium and vitamin D. Although dietary sources of both nutrients are available, most people do not receive adequate amounts for proper bone health. In addition, the heightened awareness of damaging effects of sunlight has limited vitamin D synthesis from the skin. Fortunately, supplements are available that can supply the body with amounts necessary for bone health. As reviewed, there is ample evidence that calcium and vitamin $\mathrm{D}$ alone have the ability to prevent bone loss and reduce fracture. The one caveat is that compliance must be emphasized. Clearly, with diminished compliance the protective effects of calcium and vitamin D are lost. Continued discussions with patients to promote awareness of bone health and enhance compliance with calcium and vitamin D supplements, as well as prescription drugs, should be completed. Utilizing the entire health care team can be beneficial in this quest. Office staff should understand and remind patients about bone health at key times, such as during scheduling of bone density testing. The health care provider should highlight proper nutrition and supplementation at pertinent times. For example, annual examinations are excellent times to review calcium and vitamin D intake with recommendations for supplementation reviewed. The pharmacist has a vital role in making patients aware of the need for calcium and vitamin $\mathrm{D}$ with prescription osteoporosis therapies, including proper administration to ensure absorption of bisphosphonate medications.

\section{Disclosure}

The author reports no conflicts of interest in this work.

\section{References}

Aloia JF, Vaswani A, Krall EA, et al. 1994. Calcium supplementation with and without hormone replacement therapy to prevent postmenopausal bone loss. Ann Intern Med, 120:97-103.
Avenell A, Gillespie WJ, Gillespie LD, et al. 2005. Vitamin D and vitamin $\mathrm{D}$ analogues for preventing fractures associated with involutional and post-menopausal osteoporosis. Cochrane Database Syst Rev, 3:CD000227.

Bischoff HA, Stahelin HB, Dick W, et al. 2003. Effects of vitamin D and calcium supplementation on falls: a randomized controlled trial. $J$ Bone Miner Res, 18:343-51.

Bischoff-Ferrari HA, Willett WC, Wong JB, et al. 2005. Fracture prevention with vitamin D supplementation: a meta-analysis of randomized controlled trials. JAMA, 293:2257-64.

Bischoff-Ferrari HA, Orav EJ, Dawson-Hughes B. 2006. Effect of cholecalciferal plus calcium on falling in ambulatory older men and women: a 3 year randomized controlled trial. Arch Intern Med, 166:424-30.

Black DM, Cummings SR, Karpf DB, et al.; Fracture Intervention Trial 1996. Randomized trial of effect of alendronate on risk of fracture in women with existing vertebral fractures: the Fracture Intervention Trial. FIT Research Group. Lancet, 348:1535-41.

Bo-Linn GW, Davis GR, Buddruss DJ, et al. 1984. An evaluation of the importance of gastric acid secretion in the absorption of dietary calcium. J Clin Invest, 73:640-57.

Boonen S, Lips P, Bouillon R, et al. 2007. Need for additional calcium to reduce the risk of hip fracture with vitamin D supplementation: evidence from a comparative metaanalysis of randomized controlled trials. J Clin Endocrinol Metab, 92:1415-23.

Borghi L, Schianchhi T, Meschi T, et al. 2002. Comparison of two diets for the prevention of recurrent stones in idiopathic hypercalciuria. N Engl J Med, 346:77-84.

Bouillon R. 2001. Vitamin D: from photosynthesis, metabolism, and action to clinical applications. In: DeGroot LJ, Jameson JL, (eds). Endocrinology. Philadelphia, PA: WB Saunders, pp. 1009-28.

Breslau NA. 1994. Calcium, estrogen, and progestin in the treatment of osteoporosis. Rheum Dis Clin North Am, 20:691-716.

Bringhurst FR, Demay MB, et al. 2005. Bone and mineral metabolism in health and disease. In: Harrison's Principles of Internal Medicine, 16th ed, Vol II. New York: McGraw Medical Publishing Division, pp. 2246-9.

Chapuy MC, Arlot ME, Duboeuf F, et al. 1992. Vitamin D3 and calcium to prevent hip fractures in elderly women. $N$ Engl J Med, 327:1637-42.

Chapuy MC, Pamphile R, Paris E, et al. 2002. Combined calcium and vitamin D3 supplementation in elderly women: confirmation of reversal of secondary hyperparathyroidism and hip fracture risk: the Decalyos II study. Osteoporosis Int, 13:257-64.

Cummings RG, Nevitt MC. 1997. Calcium for prevention of osteoporotic fractures in postmenopausal women. J Bone Miner Res, 12:1321-9.

Curhan GC, Willett WC, Speizer FE, et al. 1997. Comparison of dietary calcium with supplemental calcium and other nutrients as factors affecting the risk for kidney stones in women. Ann Intern Med, 126:497-504.

Daniels CE. 2001. Estrogen therapy for osteoporosis prevention in postmenopausal women. Pharmacy Update-NIH, March/April.

Dawson-Hughes B, Dallal GE, Krall EA, et al. 1990. A controlled trial of the effect of calcium supplementation on bone density in postmenopausal women. $N$ Engl J Med, 323:878-83.

Dawson-Hughes B, Harris SS, Krall EA, et al. 1995. Rates of bone loss in postmenopausal women randomly assigned to one of two dosages of vitamin D. Am J Clin Nutr, 61:1140-45.

Dawson-Hughes B, Harris SS, Krall EA, et al. 1997. Effect of calcium and vitamin D supplementation on bone density in men and women 65 years of age or older. N Engl J Med, 337:670-6.

DeLuca HF. 2004. Overview of general physiologic features and functions of vitamin D. Am J Clin Nutr, 80(Suppl):1689S-96S.

Eli Lilly. 2008. Forteo prescribing information [online]. Accessed on May 12, 2008. URL: http://pi.lilly.com/us/forteo-pi.pdf.

Gallagher JC, Riggs BL, Deluca HF. 1980. Effect of estrogen on calcium absorption and serum vitamin D metabolites in postmenopausal osteoporosis. J Clin Endocrinol Metab, 51:1359-64.

Gallagher JC, Goldgar D, Moy A. 1987. Total bone calcium in women: Effect of age and menopause status. J Bone Min Res, 2:491-6. 
Grant AM, Avenell A, et al.; RECORD Trial Group 2005. Oral vitamin D3 and calcium for secondary prevention of low-trauma fractures in elderly people (Randomised Evaluation of Calcium Or vitamin D, RECORD): a randomised placebo-controlled trial. Lancet, 365:1621-8.

Harwood RH, Sahota O, Gaynor K, et al.; The Nottingham Neck of Femur (NONOF) Study. 2004. A randomized, controlled comparison of different calcium and vitamin D supplementation regimens in elderly women after hip fracture: The NONOF study. Age Ageing, 33:45-51.

Heaney RP, Saville PD, Recker RR. 1975. Calcium absorption as a function of calcium intake. J Lab Clin Med, 85:881-90.

Heaney RP. 2006. Absorbability and utility of calcium in mineral waters. Am J Clin Nutr, 84:371-4.

Heaney RP, Dowell SD, Bierman J, et al. 2001. Absorbability and cost effectiveness in calcium supplementation. $J$ Am Coll Nutr, 20:239-46.

Holick MF. 2004a. Vitamin D: Importance and prevention of cancers, type 1 diabetes, heart disease, and osteoporosis. Am J Clin Nutr, 79:362-71.

Holick MF. 2004b. Sunlight and vitamin D for bone health and prevention of autoimmune diseases, cancers and cardiovascular disease. Am J Clin Nutr, 80(Suppl):1678S-88S.

Holick MF. 2005a. Vitamin D. In Modern nutrition in health and disease. 10th edition. Shrils M.et al. (eds). Baltimore, MA: Lippincott Williams and Wilkins, pp. 329-45.

Holick MF. 2005b. Vitamin D: important for prevention of osteoporosis, cardiovascular heart disease, type 1 diabetes, autoimmune diseases, and some cancers. South Med J, 98:1024-7.

Holick MF, Siris ES, Binkley N, et al. 2005. Prevalence of Vitamin D inadequacy among postmenopausal North American women receiving osteoporosis therapy. J Clin Endocrinol Metab, 90:3215-24.

Holick MF. 2006. Resurrection of vitamin D deficiency and rickets. J Clin Invest, 116:2062-72.

Holick MF, Biancuzzo RM, Chen TC, et al. 2008. Vitamin D2 is as effective as vitamin D3 in maintaining circulating concentrations of 25hydroxyvitamin D. J Clin Endocrinol Metab, 93:677-81.

[IOM] Institute of Medicine, Food and Nutrition Board. 1997. Dietary reference intakes: calcium, phosphorus, magnesium, vitamin D and fluoride. Washington DC: National Academy Press, p 432.

Jackson RD, LaCroix AZ, Gass M, et al. 2006. Calcium plus vitamin D supplementation and the risk of fractures. N Engl J Med, 354:669-83.

Jellin JM, Gregory P, Batz F, et al. 2000. Pharmacist's letter/prescriber's letter. Natural Medicines Comprehensive Database. 3rd edition ed. Stockton, CA: Therapeutic Research Facility.

LeBoff MS, Kohlmeier L, Hurwitz S, et al. 1999. Occult vitamin D deficiency in postmenopausal US women with acute hip fracture. JAMA, 251:1505-11.

Lips P. 2001. Vitamin D deficiency and secondary hyperparathyroidism in the elderly: consequences for bone loss and fractures and therapeutic implications. Endocr Rev, 22:477-501.

MacLaughlin JA, Anderson RR, Holick MF. 1982. Spectral character of sunlight modulates photosynthesis of previtamin D3 and its photoisomers in human skin. Science, 216:1001-3.

Moore C, Murphy MM, Keast DR, et al. 2004. Vitamin D intake in the United States. J Am Diet Assoc, 104:9980-3.

[NIH] National Institutes of Health consensus statement. 1994. Optimal calcium intake. JAMA, 272:1942-8.

[NDC] National Dairy Council. 2004. USDA's Continuing survey of food intakes by individuals 1994-1996 [online]. Accessed on March 22, 2008. URL: http://www.nationaldairycouncil.org/NationalDairy Council/Nutrition/nutrients/Calcium_Recommendations.pdf.

[NOF] National Osteoporosis Foundation. 2008. Clinicians guide to prevention and treatment of osteoporosis [online]. Accessed on March 10, 2008. URL: http://www.nof.org/professionals/NOF_Clinicians_Guide.pdf.
Nesby-O’Dell S, Scanlon KS, Cogswell ME, et al. 2002. Hypovitaminosis D prevalence and determinants among African American and white women of reproductive age: Third National Health and Nutrition Examination Survey, 1988-1994. Am J Clin Nutr, 76:187-92.

[NAMS] North American Menopause Society. 2006. Management of osteoporosis in the postmenopausal women: 2006 position statement of the North American Menopause Society. Menopause, 13:340-67.

O'Connell MD, Madden DM, Murray AM, et al. Effects of proton pump inhibitors on calcium carbonate absorption in women: A randomized crossover trial. Am J Med, 118:778-81.

Papadimitropoulos E, Wells G, Shea B, et al. 2002. Meta-analyses of therapies for postmenopausal osteoporosis. VIII: Meta-analysis of the efficacy of vitamin $\mathrm{D}$ treatment in preventing osteoporosis in postmenopausal women. Endocr Rev, 23:560-9.

Recker RR. 1985. Calcium absorption and achlorhydria. $N$ Engl J Med, 313:70-3.

Reid IR. 2003. Clinical aspects of the use of vitamin D and its metabolites. In: Orwoll ES, Bliziotes M (eds). Osteoporosis: Pathophysiology and Clinical Management. Totowa, NJ: Humana Press, pp 293-307.

Shannon MT, Wilson BA, Stang CL. 2000. Health Professionals' Drug Guide. Stamford, CT: Appleton and Lange.

Shea B, Wells G, Cranney A, et al. 2004. Calcium supplementation on bone loss in postmenopausal women. Cochrane Database Syst Rev, 1:CD004526

Snijder MB, van Schoor NM, Pluijm SM, et al. 2006. Vitamin D status in relation to one-year risk of recurrent falling in older men and women. J Clin Endocrinol Metab, 91:2980-5.

Stafford RS, Drieling RL, Johns R, et al. 2005. National patterns of calcium use in osteoporosis in the United States. J Reprod Med, 50(11 Suppl):885-90.

[SCSEDR] Standing Committee on the Scientific Evaluation of Dietary Reference Intakes. 1997. Dietary reference intakes for calcium, phosphorus, magnesium, vitamin D, and fluoride. Washington, D.C.: National Academy Press.

Sunyecz JA, Weisman SM. 2005. The role of calcium in osteoporosis drug therapy. J Womens Health, 14:180-92.

Tang BM, Eslick GD, Nowson C, et al. 2007. Use of calcium or calcium in combination with vitamin $\mathrm{D}$ supplementation to prevent fractures and bone loss in people aged 50 years and older: a meta-analysis. Lancet, 370:657-66.

Tangpricha V, Koutkia Pv, Rieke SM, et al. 2003. Fortification of orange juice with vitamin D: a novel approach to enhance vitamin D nutritional health. Am J Clin Nutr, 77:1478-83.

Thom T, Haase N, Rosamond W, et al. 2006. Heart Disease and stroke statistics - 2006 update: a report from the American Heart Association Statistics Committee and Stroke Statistics Subcommittee. Circulation, 113:85-151.

[USDH] US Department of Health and Human Services. 2004. Bone health and osteoporosis: a report of the Surgeon General.

Vieth R. 1999. Vitamin D supplementation, 25-hydroxyvitamin D concentrations and safety. Am J Clin Nutr, 69:842-56.

Weaver CM, Fleet JC. 2004. Vitamin D requirements: current and future. Am J Clin Nutr, 0(suppl):1735S-9S.

Weisman SM. 2005. Calcium connection to bone health across a woman's lifespan - A roundtable. J Reprod Med, 50(11 Suppl):879-84.

Yang YX, Lewis JD, Epstein S, et al. 2006. Long-term proton pump inhibitor therapy and risk of hip fracture. JAMA, 296:2947-53. 